\title{
UMBILICAL HYPERSURFACES IN AFFINELY CONNECTED SPACES
}

\section{CURTIS M. FULTON}

In this paper we present a definition of an umbilical hypersurface in an $m$-dimensional space $M$ of affine connection. Some properties of such hypersurfaces are found when $M$ is assumed to be projectively flat.

In general we follow the notation in [3] but adopt the positioning of indices used in [4]. Thus the usual summation convention holds. Greek indices range over the dimension of $M$, Latin indices take the values $1,2, \cdots, n=m-1$. Let $e_{\alpha}$ be a base field and $\bar{w}^{\alpha}$ the dual 1forms associated with it. We assume the connection on $M$ to be torsion-free which implies that $[3$, p. 62]

$$
0=d \bar{w}^{\alpha}+\bar{w}_{\lambda}^{\alpha} \wedge \bar{w}^{\lambda} .
$$

We also make use of the second Cartan structural equation [3, p. 63]

$$
\bar{R}_{\beta}^{\alpha}=d \bar{w}_{\beta}^{\alpha}+\bar{w}_{\lambda}^{\alpha} \wedge \bar{w}_{\beta}^{\lambda} .
$$

For any vector $V$ on $M$ we define a 1 -form $\bar{R}_{\beta}(V)$ as follows:

$$
\left(m^{2}-1\right) \bar{R}_{\beta}(V)=(m+1) \bar{R}_{\beta}^{\lambda}\left(V, e_{\lambda}\right)-\bar{R}_{\lambda}^{\lambda}\left(V, e_{\beta}\right) .
$$

On account of the first Bianchi identity which is valid because of (1) $[4$, p. 51] we find that

$$
(m+1) \bar{w}^{\lambda} \wedge \bar{R}_{\lambda}+\bar{R}_{\lambda}^{\lambda}=0 .
$$

The Weyl tensor $[1$, p. 88], [5, p. 289] is expressed by the 2-form

$$
\bar{P}_{\beta}^{\alpha}=\bar{R}_{\beta}^{\alpha}+\tau \bar{w}^{\lambda} \wedge \bar{R}_{\lambda} \delta^{\alpha}{ }_{\beta}+\tau \bar{w}^{\alpha} \wedge \bar{R}_{\beta} .
$$

Here it might be helpful to consult [6] in comparing notations. For $m>2$, the space $M$ is called projectively flat if $\bar{P}_{\beta}^{\alpha_{\beta}}=0$. This implies

$$
d \bar{R}_{\beta}+\bar{R}_{\lambda} \wedge \bar{w}_{\beta}=0 .
$$

For $m=2, \bar{P}_{\beta}$ is identically zero and a projectively flat space is then defined by (6).

In dealing with a hypersurface $N$ we let the vectors $e_{i}$ be tangent to $N$ and let $e_{m}$ be the local "normal" [1, pp. 138, 155]. Restricting (2) to vectors on $N$ we have $[3$, p. 82]

$$
\bar{R}^{i}{ }_{j}=R^{i}{ }_{j}+w_{m}^{i} \wedge w^{m}{ }_{j}
$$

Received by the editors August 18, 1967. 
Definition. A hypersurface $N$ is locally umbilical if for vectors on $N$

$$
w_{m}^{i}=w^{i} .
$$

THEOREM. An umbilical hypersurface $N$ of a projectively flat space $M$ is itself projectively flat.

Proof. Using (7), (8), (1) we infer $\bar{R}_{h}^{h}=R_{h}^{h}$. Now we rewrite $\bar{P}^{m}{ }_{m}=0$ by means of (5) and (4) and get

$$
m \bar{R}_{\lambda}^{\lambda}=(m+1) R^{h} \text {. }
$$

Also, expressing $\bar{P}^{m}{ }_{j}\left(X, e_{m}\right)=0$ with the aid of (5) leads to

$$
\bar{R}_{j}^{m_{j}}\left(X, e_{m}\right)=\bar{R}_{j}(X)
$$

for a vector $X$ belonging to $N$. Relations (7) and (8) yield

$$
\bar{R}^{h_{j}}\left(X, e_{h}\right)=R^{h}{ }_{j}\left(X, e_{h}\right)-(n-1) w^{m_{j}}(X) .
$$

If we apply the definition (3) to $N$ we conclude from (9), (10), (11) that

$$
\bar{R}_{j}=R_{j}-w^{m}{ }_{j} .
$$

Combination of (5), (7), (8), (9), (12) gives $\bar{P}^{i_{j}}=P^{\boldsymbol{i}_{j}}$. This tells us that $N$ is projectively flat if $n$ is at least 3 .

For $n=2$ we make use of (5) to change $\bar{P}_{m}^{h}\left(X, e_{h}\right)=0$ to $\bar{R}_{m}^{h}\left(X, e_{h}\right)$ $-(n-1) \bar{R}_{m}(X)=0$. On the other hand, (2) in conjunction with (8) and (1) becomes $\bar{R}_{m}^{h}\left(X, e_{h}\right)+(n-1) w_{m}^{m}(X)=0$. By comparison,

$$
w_{m}^{m}=-\bar{R}_{m} \text {. }
$$

Moreover, $\bar{P}_{j}{ }_{j}=0$ gives rise to $\bar{R}^{m_{j}}=0$ for vectors on $N$. Hence, in accordance with (2)

$$
d w^{m}{ }_{j}+w^{m_{\lambda}} \wedge w^{\lambda}{ }_{j}=0 .
$$

From (12), (13), (14) we see that

$$
d \bar{R}_{j}+\bar{R}_{\lambda} \wedge w^{\lambda}{ }_{j}=d R_{j}+R_{h} \wedge w^{h}{ }_{j} .
$$

Owing to the definition (6) of a projectively flat 2-space, this completes the proof.

In what follows let $X, Y$ be vectors on $N$, and $Z$ be the normal. The definition (8) now reads: $D_{X} Z=X$. Thus,

$$
\bar{D}_{X} Z=X-g(Z, X) Z,
$$

where $g$ is seen to be linear in the second slot. Actually, it would be 
more precise to say that $N$ is umbilical with respect to the normal $Z$. We now think of $N$ as one of a family of hypersurfaces and postulate that (15) is valid when $X$ is taken equal to $Z$. In this case $\bar{D}_{Z} Z$ is proportional to $Z$ and the trajectories of the family whose tangent vector is $Z$ are geodesics. Thus, we have a fanily of parallel umbilical hypersurfaces [2]. Such hypersurfaces exist when solutions $Z$ of (15) can be found for arbitrary $X$. The situation is elucidated by the following.

Theorem. For $m>2$, equations (15) are completely integrable if and only if $M$ is projectively flat and $g(Z, X)=\bar{w}^{\lambda}(Z) \bar{R}_{\lambda}(X)$.

Proof. In view of (1) the integrability conditions of (15) may be written $[3$, p. 59]

$$
\begin{aligned}
\bar{R}(X, Y) Z= & -g(Z, Y) X+g(Z, X) Y \\
& -\{X g(Z, Y)-Y g(Z, X)-g(Z,[X, Y])\} Z .
\end{aligned}
$$

If we first assume that solutions of (15) exist in all directions, the first Bianchi identity may be applied to 3 independent vectors $X, Y, Z$, $m$ being greater than 2. This allows for a modified form of (16), namely

$$
\bar{R}(X, Y) Z=-g(Z, Y) X+g(Z, X) Y-\{g(X, Y)-g(Y, X)\} Z .
$$

We notice at once that $g$ must be linear in the first slot also and make $Z=e_{\beta}$ for simplification. Furthermore, setting $g\left(e_{\beta}, X\right)=g_{\beta}(X),(17)$ appears in the form

$$
\bar{R}^{\alpha}{ }_{\beta}=-\bar{w}^{\alpha} \wedge g_{\beta}-\bar{w}^{\lambda} \wedge g_{\lambda} \delta^{\alpha}{ }_{\beta} .
$$

From (3) it is now easily seen that $\bar{R}_{\beta}=g_{\beta}$ and (5) gives the desired result, $\bar{P}_{\beta}^{\alpha_{\beta}}=0$. In addition, $g(Z, X)=\bar{w}^{\lambda}(Z) \bar{R}_{\lambda}(X)$.

For the converse we write (15)

$$
\bar{w}_{\beta}^{\lambda}=\bar{w}^{\lambda}-\bar{R}_{\beta} \delta^{\lambda} .
$$

Similarly, the integrability conditions (16) become

$$
\bar{R}^{\alpha}{ }_{\beta}=-\bar{w}^{\alpha} \wedge \bar{R}_{\beta}-d \bar{R}_{\beta} \delta^{\alpha}{ }_{\beta} .
$$

But now $\bar{P}_{\beta}=0$, implying that

$$
\bar{R}^{\alpha}{ }_{\beta}=-\bar{w}^{\alpha} \wedge \bar{R}_{\beta}-i \bar{c}^{\lambda} \wedge \bar{R}_{\lambda} \delta^{\alpha}{ }_{\beta} .
$$

Because of (18) we find that $\bar{R}_{\lambda} \wedge \bar{w}_{\beta}^{\lambda}=-\bar{w}^{\lambda} \wedge \bar{R}_{\lambda}$ and then, since (6) holds, (19) is a consequence of (20).

Lastly, from the preceding material the reader may supply the proof of the 
THEOREM. For $m=2$, equations (18) are completely integrable if and only if $M$ is projectively flat.

ADDEndum. In comparison with spaces of constant curvature [2], one might be inclined to multiply the vector $e_{m}$ occurring in the definition (8) by some factor. This, however, would not materially alter our proofs because of the absence of a metric. Similarly, we could use the additional definition $w^{m}{ }_{m}=0$ for vectors on $N$, which means that the derivative of $e_{m}$ is tangent to $N$. Then, on account of (1), $w^{m}{ }_{h} \wedge w^{h}=0$, and owing to the definition (8) we infer from (2) that $\bar{R}^{m_{m}}=0$. Since $\bar{P}_{m}^{m_{m}}=0$, (5) and (4) now tell us that $\bar{R}_{\lambda}^{\lambda_{\lambda}}=0$, and because of (9), we also find $R_{h}^{h}=0$. This means that the connection of $N$ preserves volume [5, p. 144].

Acknowledgment. The author is indebted to the referee for the addendum.

\section{REFERENCES}

1. L. P. Eisenhart, Non-Riemannian geometry, Amer. Math. Soc. Colloq. Publ., Vol. 8, Amer. Math. Soc., Providence, R. I., 1922; reprint 1964.

2. C. M. Fulton, Parallel umbilical hypersurfaces, Proc. Amer. Math. Soc. 16 (1965), 1039-1041.

3. N. J. Hicks, Notes on differential geometry, Van Nostrand, New York, 1965.

4. M. M. Postnikov, The variational theory of geodesics, Saunders, Philadelphia, Pa., 1967.

5. J. A. Schouten, Ricci-calculus, Springer-Verlag, Berlin, 1954.

6. J. D. Zund, Koszul's formalism and the Ricci calculus, Tensor 18 (1967), 150161.

University of California, Davis 